\title{
A NEW VERSION OF THE TWO-DIMENSIONAL LAX-FRIEDRICHS SCHEME
}

\author{
T. BOUKADIDA AND A. Y. LEROUX
}

\begin{abstract}
We develop a new two-dimensional version of the Lax-Friedrichs scheme, which corresponds exactly to a transport projection method. The scheme we obtain in this way is different from the one derived by averaging the one-dimensional scheme in the two directions as usually done. The LaxFriedrichs scheme is known to be a very stable scheme with much diffusion. However, this diffusion can be easily reduced by using corrected fluxes, without altering the total-variation estimates. The accuracy of this corrected scheme is of order two except near a local extrema. The numerical results computed by using this corrected scheme are similar to the ones obtained by using the Godunov scheme with corrected fluxes but require less CPU time. Convergence towards the entropy solution is proved, and some extensions to systems of conservation laws or three-dimensional models are discussed. Some numerical experiments are reported.
\end{abstract}

\section{INTRODUCTION}

We are concerned with the approximation of the initial value problem in two space dimensions, on $Q_{T}=\mathbb{R}^{2} \times(0, T)$ for a given $T>0$,

$$
\begin{gathered}
u_{t}+f(u)_{x}+g(u)_{y}=0 \text { for }(x, y, t) \in Q_{T}, \\
u(x, y, 0)=u_{0}(x, y) \text { for }(x, y) \in \mathbb{R}^{2},
\end{gathered}
$$

where $u$ is a function of $(x, y, t)$ defined on $Q_{T}$. We assume that

$$
f, g \in C^{2}(\mathbb{R}), \quad u_{0} \in L^{\infty}\left(\mathbb{R}^{2}\right) \cap \mathrm{BV}_{\text {loc }}\left(\mathbb{R}^{2}\right),
$$

where $B V_{\text {loc }}\left(\mathbb{R}^{2}\right)$ is the set of all locally bounded variation functions on $\mathbb{R}^{2}$, in the sense of Tonelli-Cesari. This means that for any bounded open set $\Omega$ in $\mathbb{R}^{2}$,

$$
\operatorname{Var}(u ; \Omega)=\int_{\mathbf{R}} \operatorname{Var}\left(u(\cdot, y) ; \Omega_{y}\right) d y+\int_{\mathbf{R}} \operatorname{Var}\left(u(x, \cdot) ; \Omega_{x}\right) d x
$$

with

$$
\boldsymbol{\Omega}_{y}=\Omega \cap\{(\xi, y) ; \xi \in \mathbb{R}\} ; \quad \Omega_{x}=\Omega \cap\{(x, \eta) ; \eta \in \mathbb{R}\} .
$$

The entropy condition, which is required for uniqueness, is contained in the following Kružkov characterization (see [8]).

Received by the editor April 20, 1990 and, in revised form, December 30, 1991 and September 20, 1993.

1991 Mathematics Subject Classification. Primary 35L65, 65M12.

(C) 1994 American Mathematical Society $0025-5718 / 94 \$ 1.00+\$ .25$ per page 
For any real $k$ and any nonnegative test function $\phi$ lying in $C^{2}\left(\mathbb{R}^{2} \times[0, T)\right)$,

$$
\begin{aligned}
& \iiint_{Q_{T}}(\mid u- k \mid \phi_{t}+\operatorname{sgn}(u-k) \\
& \times\left[(f(u)-f(k)) \phi_{x}+(g(u)-g(k)) \phi_{y}\right] d x d y d t \\
& \quad+\iint_{\mathbb{R}^{2}}\left|u_{0}-k\right| \phi(x, y, 0) d x d y \geq 0 .
\end{aligned}
$$

Here sgn denotes the sign function. The formulation (4) leads to the definition of a semigroup operator $S(t)$ such that $u(\cdot, t)=S(t) u_{0}$ for all $t \in(0, T]$. Such a solution is called a Kružkov solution or an entropy solution. We know (see [7]) that this semigroup is a contraction in $L^{1}\left(\mathbb{R}^{2}\right)$.

The usual version of the Lax-Friedrichs scheme for several space dimensions has been developed in [9], and convergence is proved in [3]. This scheme is obtained as an average of one-dimensional schemes proceeding in each direction. Even though the one-dimensional scheme is really a transport projection method, that is, a combination of $S(t)$-operators and projection operators on a discrete space, this is no longer true for the several-dimensional scheme constructed as above. Moreover, the dimensional averaging introduces an additional error on the numerical fluxes, which makes the correction of these fluxes more difficult to handle.

For each time cycle, the one-dimensional scheme corresponds to an exact computation of the fluxes from a constant piecewise approximate solution, and then to a projection on a shifted mesh. This projection introduces a great deal of linear diffusion, which can be reduced easily and efficiently. We use here the same arguments: exact computation of fluxes and shifted projections.

This construction is detailed in $\S 2$. Then $\S 3$ deals with a result of convergence involving a stability condition which is weaker than for the usual twodimensional Lax-Friedrichs scheme. The accuracy may be improved too, by using some flux-corrected method. Two such methods are proposed in $\S 4$. The first preserves the $L^{\infty}$ stability, but not the BV estimate, and leads to a really improved approximate solution. The second preserves the BV stability, but is not really efficient for the correction and involves some squeezing conditions, which makes it inoperative because the order-two accuracy is attained for too few points. Section 5 deals with a discussion of some extensions of this scheme to systems of conservation laws or to the three-dimensional case. Numerical results are given in $\S \S 2$ and 4 , to illustrate the properties of the different schemes presented in this study.

\section{THE CONSTRUCTION OF THE SCHEME}

We denote by $h=\Delta x$ the space meshsize in the $x$-and $y$-directions, and we set for any $i, j$ in $\mathbb{Z}, x_{i}=i h, y_{j}=j h$. We use for simplicity the same space meshsize in the two directions; the generalization to different meshsizes is obvious. We shall use the notations $\alpha=i+1 / 2$ and $\beta=j+1 / 2$ as a convention. In this way the plane $\mathbb{R}^{2}$ is discretized with the cells

$$
\Gamma_{\alpha \beta}=\left(x_{i}, x_{i+1}\right) \times\left(y_{j}, y_{j+1}\right)
$$

for $i, j \in \mathbb{Z}$. We define the discrete space

$$
V_{h}=\left\{v \in L^{\infty}\left(\mathbb{R}^{2}\right) \mid v=\text { constant on } \Gamma_{\alpha \beta}, i, j \in \mathbb{Z}\right\} .
$$


The constant value of $v \in V_{h}$ on the cell $\Gamma_{\alpha \beta}$ is denoted $v_{\alpha \beta}$.

Let $P_{h}$ be the orthogonal projection on $V_{h}$ associated with the usual $L^{2}$ inner product. This projection gives the usual mean value on each cell as follows:

$$
\forall v \in L^{\infty}\left(\mathbb{R}^{2}\right) \quad P_{h} v(x, y)=\frac{1}{h^{2}} \iint_{\Gamma_{\alpha \beta}} v(\xi, \eta) d \xi d \eta \quad \text { if }(x, y) \in \Gamma_{\alpha \beta} .
$$

The midpoint of the cell $\Gamma_{\alpha \beta}$ is denoted $\left(x_{\alpha}, y_{\beta}\right)$.

We construct a second discrete space, which corresponds to the shifted mesh. We set as before $\Gamma_{i j}=\left(x_{\alpha-1}, x_{\alpha}\right) \times\left(y_{\beta-1}, y_{\beta}\right)$, for $i, j \in \mathbb{Z}$, and

$$
W_{h}=\left\{w \in L^{\infty}\left(\mathbb{R}^{2}\right) \mid w \text { constant on } \Gamma_{i j}, i, j \in \mathbb{Z}\right\} .
$$

The constant value of $w \in W_{h}$ on $\Gamma_{i j}$ is denoted $w_{i j}$. The space $W_{h}$ is made up of the translated functions of $V_{h}$ with the shift $h / 2$ in both directions. The orthogonal projection onto $W_{h}$ is denoted $Q_{h}$.

Let $\Delta t$ be the time stepsize. We introduce the sequence $t_{n}=n \Delta t$, and define the ratio $r=\Delta t / h$, which will be used to control the stability.

The approximate solution at time $t_{n}$ is denoted $u^{n}$, and is supposed to belong to $V_{h}$. The numerical scheme corresponds to the following formulation:

$$
u^{n}=P_{h} S\left(\frac{\Delta t}{2}\right) Q_{h} S\left(\frac{\Delta t}{2}\right) u^{n-1} .
$$

This formula corresponds effectively to a double time cycle, each part of which advances in time by $\Delta t / 2$. We set $\mu=n+1 / 2$, and $t_{\mu}=t_{n-1}+\Delta t / 2$.

Let $n \in \mathbb{N}, n \geq 1$. We assume that the approximate solution $u^{n-1} \in V_{h}$ is known. Then by integrating over $\Gamma_{\alpha \beta} \times\left(t_{n-1}, t_{\mu}\right)$ the solution of (1) which corresponds to the Cauchy data

$$
u\left(x, y, t_{n-1}\right)=u^{n-1}(x, y)
$$

for $(x, y) \in \mathbb{R}^{2}$ at time $t=t_{n-1}$, we get the following value:

(6) $u_{\alpha \beta}^{\mu}=\frac{1}{4}\left(u_{i, j}^{n-1}+u_{i, j+1}^{n-1}+u_{i+1, j}^{n-1}+u_{i+1, j+1}^{n-1}\right)-\Phi_{\alpha, j}^{\mu}+\Phi_{\alpha, j+1}^{\mu}-\Phi_{i, \beta}^{\mu}+\Phi_{i+1, \beta}^{\mu}$, where $u_{\alpha \beta}^{\mu}$ is the averaged value of the solution at $t=t_{\mu}$ on $\Gamma_{\alpha \beta}$, and $\Phi_{\alpha j}^{\mu}$ denotes the flux through the interface

$$
\Gamma_{\alpha j}^{\mu}=\left\{(x, y, t) \mid x_{i}<x<x_{i+1}, y=y_{j}, t_{n-1}<t<t_{\mu}\right\},
$$

which is a face of $\Gamma_{i j} \times\left(t_{n-1}, t_{\mu}\right)$. The fluxes $\Phi_{\alpha, j+1}^{\mu}$ through $\Gamma_{\alpha, j+1}^{\mu}, \Phi_{i \beta}^{\mu}$ through $\Gamma_{i \beta}^{\mu}$, and $\Phi_{i+1, \beta}^{\mu}$ through $\Gamma_{i+1, \beta}^{\mu}$ are defined the same way, with

$$
\Gamma_{i \beta}^{\mu}=\left\{(x, y, t) \mid x=x_{i}, y_{j}<y<y_{j+1}, t_{n-1}<t<t_{\mu}\right\} .
$$

Figure 1 (next page) corresponds to the construction of the scheme.

We have

$$
\Phi_{\alpha j}^{\mu}=\frac{1}{h^{2}} \iint_{\Gamma_{\alpha j}^{\mu}} g(u) d y d t, \quad \Phi_{i \beta}^{\mu}=\frac{1}{h^{2}} \iint_{\Gamma_{i \beta}^{\mu}} f(u) d x d t .
$$

If $\Delta t$ is small enough (the size will be limited by a stability condition in $\S 3$ ), the exact computation of these fluxes can be performed by using a one-dimensional Riemann solver. As a matter of fact, we have

$$
\Phi_{i \beta}^{\mu}=\frac{1}{h^{2}} \int_{t_{n-1}}^{t_{\mu}} \int_{y_{j}}^{y_{j+1}} f(v(y, t)) d y d t,
$$




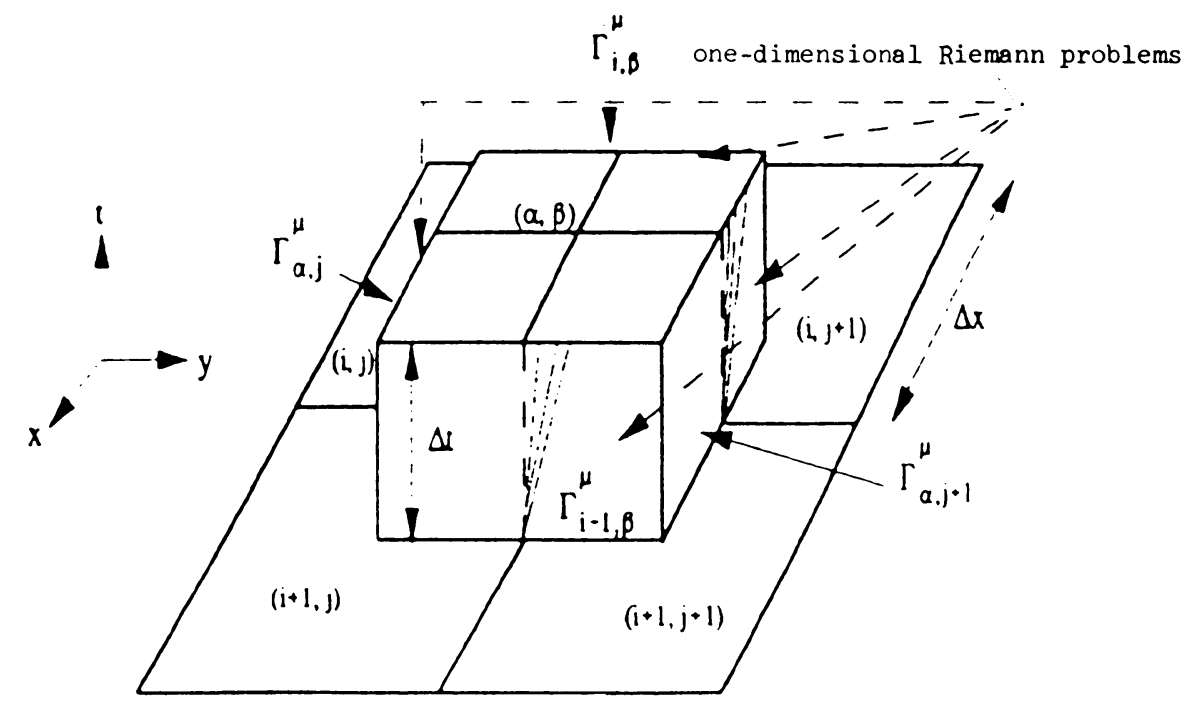

FigURE 1

where $v$ is the solution of the following Riemann problem:

$$
\begin{aligned}
& v_{t}+g(v)_{y}=0 \text { for } t>t_{n-1}, y \in \mathbb{R}, \\
& v\left(y, t_{n-1}\right)= \begin{cases}u_{i j}^{n-1} & \text { for } y<\beta h, \\
u_{i, j+1}^{n-1} & \text { for } y>\beta h .\end{cases}
\end{aligned}
$$

We compute (8) as follows. Let $\tilde{g}$ be either the convex hull of $g$ on the interval

$$
\left[u_{i j}^{n-1}, u_{i, j+1}^{n-1}\right] \text { if } u_{i j}^{n-1} \leq u_{i, j+1}^{n-1},
$$

or the concave hull of $g$ on the interval

$$
\left[u_{i, j+1}^{n-1}, u_{i j}^{n-1}\right] \text { if } u_{i j}^{n-1} \geq u_{i, j+1}^{n-1} .
$$

For instance, in the first case one gets, with $\xi=y-y_{j}, \tau=t-t_{n-1}$, that

$$
v(\xi, \tau)= \begin{cases}u_{i j}^{n-1} & \text { for } \xi \leq \tau \tilde{g}^{\prime}\left(u_{i j}^{n-1}\right), \\ {\left[\tilde{g}^{\prime}\right]^{-1}\left(\frac{\xi}{\tau}\right)} & \text { for } \tau \tilde{g}^{\prime}\left(u_{i j}^{n-1}\right)<\xi<\tau \tilde{g}^{\prime}\left(u_{i, j+1}^{n-1}\right), \\ u_{i, j+1}^{n-1} & \text { for } \xi \geq \tau \tilde{g}^{\prime}\left(u_{i, j+1}^{n-1}\right) .\end{cases}
$$

This formula takes into account the discontinuities of $v$, which correspond to linear segments of the convex (or concave) hull $\tilde{g}$.

By using (10) in (8), we get, since $\Delta t=r h$,

$$
\Phi_{i \beta}^{\mu}=\frac{r}{4}\left(1+\frac{r}{2} \tilde{g}^{\prime}\left(u_{i, j}^{n-1}\right)\right) f\left(u_{i, j}^{n-1}\right)+\frac{r}{4}\left(1-\frac{r}{2} \tilde{g}^{\prime}\left(u_{i, j+1}^{n-1}\right)\right) f\left(u_{i, j+1}^{n-1}\right)+K_{i \beta}^{\mu}
$$

with

$$
K_{i \beta}^{\mu}=\frac{1}{h^{2}} \int_{0}^{\Delta t / 2} \int_{\tau \tilde{g}^{\prime}\left(u_{i, j}^{n-1}\right)}^{\tau \tilde{g}^{\prime}\left(u_{i, j+1}^{n-1}\right)} f(v(\xi, \tau)) d \xi d \tau
$$


In this last integral we change the variable $\xi$ into $v$ by writing $\xi=\tau \tilde{g}^{\prime}(v)$, and we get

$$
K_{i \beta}^{\mu}=\frac{1}{h^{2}} \int_{0}^{\Delta t / 2} \tau d \tau \int_{\tau \tilde{g}^{\prime}\left(u_{i, j}^{n-1}\right)}^{\tau \tilde{g}^{\prime}\left(u_{i, j+1}^{n-1}\right)} f(v) \frac{d}{d v}\left(\tilde{g}^{\prime}(v)\right) d \xi .
$$

Then, by integrating by parts, we obtain

$$
K_{i \beta}^{\mu}=\frac{r^{2}}{8}\left(f\left(u_{i, j+1}^{n-1}\right) \tilde{g}^{\prime}\left(u_{i, j+1}^{n-1}\right)-f\left(u_{i j}^{n-1}\right) \tilde{g}^{\prime}\left(u_{i j}^{n-1}\right)-\int_{u_{i, j}^{n-1}}^{u_{i, j+1}^{n-1}} f^{\prime}(v) \tilde{g}^{\prime}(v) d v\right),
$$

which allows us to write $\Phi_{i \beta}^{\mu}$ the following way:

$$
\Phi_{i \beta}^{\mu}=\frac{r}{4}\left[f\left(u_{i j}^{n-1}\right)+f\left(u_{i, j+1}^{n-1}\right)\right]-\frac{r^{2}}{8} F_{i, \beta}^{n-1},
$$

where

$$
F_{i, \beta}^{n-1}=F\left(u_{i j}^{n-1}, u_{i, j+1}^{n-1}\right)
$$

with

$$
F(a, b)=\int_{a}^{b} f^{\prime}(\xi) \tilde{g}^{\prime}(\xi) d \xi .
$$

By generating the $\Phi_{\alpha j}^{\mu}$ using the same technique, we can compute the values $u_{\alpha \beta}^{\mu}$ by means of (6) for any $\alpha$ and $\beta$. This corresponds to the result of the projection of $S(\Delta t / 2) u^{n-1}$ on $W_{h}$. That is exactly the value of $Q_{h} S(\Delta t / 2) u^{n-1}$ on each $\Gamma_{\alpha \beta}$. We have computed in this way $u^{\mu} \in W_{h}$. We have

$$
\begin{aligned}
u_{\alpha, \beta}^{\mu}= & \frac{1}{4}\left[u_{i, j}^{n-1}+u_{i+1, j}^{n-1}+u_{i, j+1}^{n-1}+u_{i+1, j+1}^{n-1}\right] \\
& -\frac{r}{4}\left[f\left(u_{i+1, j}^{n-1}\right)-f\left(u_{i, j}^{n-1}\right)+f\left(u_{i+1, j+1}^{n-1}\right)-f\left(u_{i, j+1}^{n-1}\right)\right] \\
& -\frac{r}{4}\left[g\left(u_{i, j+1}^{n-1}\right)-g\left(u_{i, j}^{n-1}\right)+g\left(u_{i+1, j+1}^{n-1}\right)-g\left(u_{i+1, j}^{n-1}\right)\right] \\
& +\frac{r^{2}}{8}\left[G_{\alpha, j+1}^{n-1}-G_{\alpha, j}^{n-1}+F_{i+1, \beta}^{n-1}-F_{i, \beta}^{n-1}\right],
\end{aligned}
$$

where, as in (12),

$$
G_{\alpha, j}^{n-1}=G\left(u_{i j}^{n-1}, u_{i+1, j}^{n-1}\right), \quad \text { with } G(a, b)=\int_{a}^{b} g^{\prime}(\xi) \tilde{f}^{\prime}(\xi) d \xi,
$$

which involves a Riemann problem for the equation

$$
v_{t}+f(v)_{x}=0 \text {. }
$$

From these values we compute

$$
u^{n}=P_{h} S\left(\frac{\Delta t}{2}\right) u^{\mu}
$$

which corresponds to the same scheme as above, but with shifted indices: $i$ (resp. $j$ ) becomes $\alpha$ (resp. $\beta$ ), and $\alpha-1$ (resp. $\beta-1$ ) becomes $i$ (resp. $j$ ). This completes the construction of the scheme. 
We may notice that the computation of $F(a, b)$ or $G(a, b)$ is rather simple in practice. For example, for $g$ concave, we have

$$
F(a, b)=\frac{[f(b)-f(a)][g(b)-g(a)]}{b-a} .
$$

Moreover, by writing $g=0$, we get a one-dimensional problem (we identify all the values with the same index $i$ ), and the corresponding scheme is exactly the one-dimensional Lax-Friedrich scheme

$$
u_{\alpha}^{\mu}=\frac{1}{2}\left[u_{i}^{n-1}+u_{i+1}^{n-1}\right]=\frac{r}{2}\left[f\left(u_{i+1}^{n-1}\right)-f\left(u_{i}^{n-1}\right)\right] .
$$

The two-dimensional extension which is proposed in [3] by Conway and Smoller corresponds to (13) with $F \equiv G \equiv 0$ and after two $\Delta t / 2$-timesteps. These terms $F$ and $G$ correspond to a crossed derivative of the second order. This remark shows that this scheme is not an alternating direction scheme.

One may notice that this term allows the timestep to be twice as big in the stability condition. For example, in the linear case, with $f(u)=A u$ and $g(u)=$ $B u$, the scheme reads

$$
\begin{aligned}
u_{\alpha \beta}^{\mu}=\frac{1}{4}\left\{u_{i j}^{n-1}(1+r A)\right. & (1+r B)+u_{i, j+1}^{n-1}(1+r A)(1-r B) \\
& \left.+u_{i+1, j}^{n-1}(1-r A)(1+r B)+u_{i+1, j+1}^{n-1}(1-r A)(1-r B)\right\},
\end{aligned}
$$

which is obviously $L^{\infty}$-stable for $r|A| \leq 1$ and $r|B| \leq 1$. If we get rid of the $r^{2}$ terms in the expression above, the condition becomes $r(|A|+|B|) \leq 1$, and the scheme becomes the usual Lax-Friedrichs scheme (after two $\Delta t / 2$-timesteps).

We present now a numerical result using the scheme (13) and the flux functions

$$
f(u)=g(u)=\frac{u^{2}}{2}
$$

and the initial data

$$
u(x, y, 0)= \begin{cases}1 & \text { if } 0<x<0.45 \\ 0 & \text { elsewhere }\end{cases}
$$

In Figure 2 we have used a $40 \times 40$ space grid and a CFL number equal to 0.8 .

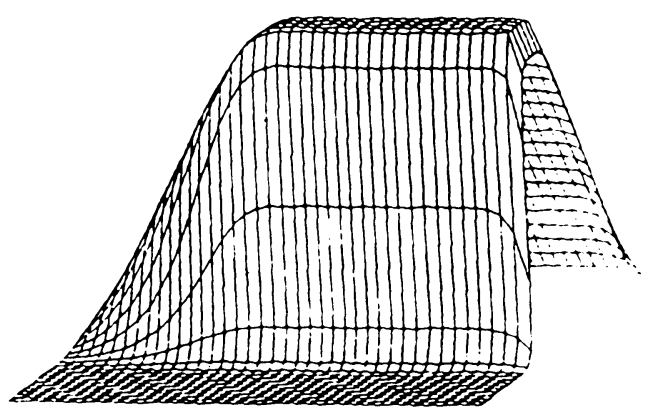

FIGURE 2 
Some numerical diffusion of the shock surface is still present, but less than for the usual scheme. The aim of $\S 4$ is to reduce this numerical diffusion.

\section{A CONVERGENCE THEOREM}

We consider the following form of the scheme:

$$
\begin{aligned}
& u^{n}=P_{h} S\left(\frac{\Delta t}{2}\right) Q_{h} S\left(\frac{\Delta t}{2}\right) u^{n-1}, \\
& u^{0}=P_{h} u_{0} .
\end{aligned}
$$

The convergence theorem is now easy to prove by using the properties of $S$, $P_{h}$, and $Q_{h}$. However, we have to notice that the practical interest of (15) is in its potential to provide an effective computational scheme. Actually, this is possible only when $\Delta t$ is small enough to prevent too complex interactions between the waves emanating from the different cells. Such interactions cannot occur if the Courant-Friedrichs-Lewy (CFL) condition

$$
\frac{\Delta t}{h} \underset{\left\{|\xi| \leq\left|u_{0}\right|_{L \infty}\right\}}{\operatorname{Max}}\left(\left|g^{\prime}(\xi)\right|,\left|f^{\prime}(\xi)\right|\right) \leq 1
$$

is satisfied. This comes from the following argument. The solution of (1) computed by starting from $u^{n-1}$ as the Cauchy data at $t=t_{n-1}$ coincides with the solution of a Riemann problem such as (9) on each side of $\Gamma_{i, j} \times\left[t_{n-1}, t_{\mu}\right]$ as long as $\Delta t$ satisfies (16). Otherwise, the solution on the segments $\Gamma_{\alpha j}^{\mu}$ or $\Gamma_{i \beta}^{\mu}$ will be perturbed by characteristics coming from cells other than the adjacent one.

We underline the importance in the introduction of $F$ and $G$, which allows the CFL condition (16) with a bound (also called CFL number) equal to 1 instead of $1 / 2$ as usual for two-dimensional schemes, including the usual version of the two-dimensional Lax-Friedrichs scheme.

Theorem 1. The formula (15) allows to construct a sequence of approximate solutions which is convergent towards the Kružkov solution of (1). Moreover, if the CFL condition (16) is satisfied, a numerical scheme can be derived from (15) and leads to convergence.

The proof is part of another one given in $\S 4$ below. We only mention that it is based on properties of $L^{1}$-contraction and $L^{\infty}$ and BV stability of the operators $S(t), P_{h}$, and $Q_{h}$.

\section{ANTIDIFFUSION}

The main drawback of the Lax-Friedrichs scheme is its excessive numerical diffusion. If we are able to reduce efficiently this diffusion, we get a scheme for which the error is lower than for many other schemes. We have noticed that the main part of the diffusion comes from the double projection. This part of the diffusion can easily be exhibited and the corresponding correction easily performed. This is evaluated by applying the scheme to the trivial equation 
$u_{t}=0$. We get

$$
\begin{aligned}
u_{i, j}^{n}= & u_{i, j}^{n-1}+\frac{1}{8}\left(u_{i+1, j}^{n-1}-2 u_{i, j}^{n-1}+u_{i-1, j}^{n-1}\right)+\frac{1}{8}\left(u_{i, j+1}^{n-1}-2 u_{i, j}^{n-1}+u_{i, j-1}^{n-1}\right) \\
& +\frac{1}{16}\left(u_{i+1, j+1}^{n-1}-2 u_{i, j}^{n-1}+u_{i-1, j-1}^{n-1}\right) \\
& +\frac{1}{16}\left(u_{i+1, j-1}^{n-1}-2 u_{i, j}^{n-1}+u_{i-1, j+1}^{n-1}\right)
\end{aligned}
$$

This corresponds to a discretization of a heat equation using four directions in the plane: the two axes and the two diagonals. By using Taylor expansion, we get

$$
u_{i, j}^{n}=u_{i, j}^{n-1}+\frac{h^{2}}{4}\left(\Delta u^{n-1}\right)_{i, j}+o\left(h^{2}\right),
$$

where $\left(\Delta u^{n-1}\right)_{i, j}$ denotes the value of the Laplace operator applied to $u^{n}$ at the point $(i h, j k)$. From this formula we get the amount of diffusion introduced at each double cycle, and the antidiffusion technique is now a way to get rid of it.

The new version of the scheme corresponds to the following formulas. First we compute the value $\bar{u}^{n}$, using the scheme (15) as before. Then we perform the antidiffusion step by using some operator $A_{h}$; that is,

$$
u^{n}=A_{h} \bar{u}^{n} \text {. }
$$

This leads to the following scheme:

$$
u^{n}=A_{h} P_{h} S\left(\frac{\Delta t}{2}\right) Q_{h} S\left(\frac{\Delta t}{2}\right) u^{n-1} .
$$

The operator $A_{h}$ has the form

$$
u_{i, j}^{n}=\bar{u}_{i, j}^{n}-a_{\alpha, j}^{n}+a_{\alpha-1, j}^{n}-a_{i, \beta}^{n}+a_{i, \beta-1}^{n} .
$$

The coefficients $a_{\alpha, j}^{n}$ or $a_{i, \beta}^{n}$ are real parameters called corrected fluxes. Such methods were introduced in [1] and analyzed, for example, in [10].

The corrected fluxes correspond to space finite differences because our aim is to approximate a Laplace operator.

They are written this way:

$$
\begin{aligned}
& a_{\alpha, j}^{n}=\mu_{\alpha, j}^{n}\left(\bar{u}_{i+2, j}^{n}-\bar{u}_{i+1, j}^{n}\right)=\lambda_{\alpha, j}^{n}\left(\bar{u}_{i, j}^{n}-\bar{u}_{i-1, j}^{n}\right), \\
& a_{i, \beta}^{n}=\mu_{i, \beta}^{n}\left(\bar{u}_{i, j+2}^{n}-\bar{u}_{i, j+1}^{n}\right)=\lambda_{i, \beta}^{n}\left(\bar{u}_{i, j}^{n}-\bar{u}_{i, j-1}^{n}\right),
\end{aligned}
$$

with some real parameters $\mu_{\alpha, j}^{n}, \mu_{i, \beta}^{n}, \lambda_{\alpha, j}^{n}, \lambda_{i, \beta}^{n}$ to be chosen later. We first look for sufficient conditions concerning these parameters.

Lemma 1. If for any $i, j$

$$
\begin{array}{cl}
\mu_{\alpha, j}^{n} \geq 0, \quad \mu_{i, \beta}^{n} \geq 0, & \lambda_{\alpha, j}^{n} \geq 0, \quad \lambda_{i, \beta}^{n} \geq 0, \\
\mu_{\alpha-1, j}^{n}+\lambda_{\alpha, j}^{n} \leq \frac{1}{2}, & \lambda_{i, \beta}^{n}+\mu_{i, \beta-1}^{n} \leq \frac{1}{2},
\end{array}
$$

then the scheme (19) is $L^{\infty}$-stable, that is,

$$
\left|u^{n}\right|_{L^{\infty}} \leq\left|u^{n-1}\right|_{L^{\infty}}
$$


Proof. The $L^{\infty}$ stability of $S(t), P_{h}$, or $Q_{h}$ implies that $\left|\bar{u}^{n}\right|_{L^{\infty}} \leq\left|u^{n-1}\right|_{L^{\infty}}$. Next, for any $i$ and $j$, we have

$$
\begin{aligned}
u_{i, j}^{n}= & \left(\frac{1}{2}-\lambda_{\alpha, j}^{n}-\mu_{\alpha-1, j}^{n}\right) \bar{u}_{i, j}^{n}+\lambda_{\alpha, j}^{n} \bar{u}_{i-1, j}^{n}+\mu_{\alpha-1, j}^{n} \bar{u}_{i+1, j}^{n} \\
& +\left(\frac{1}{2}-\lambda_{i, \beta}^{n}-\mu_{i, \beta-1}^{n}\right) \bar{u}_{i, j}^{n}+\lambda_{i, \beta}^{n} \bar{u}_{i, j-1}^{n}+\mu_{i, \beta-1}^{n} \bar{u}_{i, j+1}^{n},
\end{aligned}
$$

where all coefficients are nonnegative. Then $L^{\infty}$ stability is easily derived.

Of course, the conditions (22) are not sufficient to define uniquely the $a_{\alpha, j}^{n}$ and $a_{i, \beta}^{n}$. In practice, we consider (22) as a set of constraints, and the optimal scheme is obtained by minimizing

$$
\left|a_{\alpha, j}^{n}-\left(u_{i+1, j}^{n}-u_{i, j}^{n}\right)\right|^{2} \text { and }\left|a_{i, \beta}^{n}-\left(u_{i, j+1}^{n}-u_{i, j}^{n}\right)\right|^{2}
$$

subject to the constraints (22). Other choices are also possible, for example, by computing successively

$$
\begin{aligned}
\bar{\sigma}_{\alpha j}^{n}= & \operatorname{sgn}\left(\bar{u}_{i+1, j}^{n}-\bar{u}_{i j}^{n}\right), \\
\bar{a}_{\alpha j}^{n}= & \operatorname{Max}\left(0, \min \left\{\bar{\sigma}_{\alpha j}^{n}\left(\bar{u}_{i+2, j}^{n}-\bar{u}_{i+1, j}^{n}\right),\right.\right. \\
& \left.\left.\frac{1}{2}\left|\bar{u}_{i+1, j}^{n}-\bar{u}_{i j}^{n}\right|, \bar{\sigma}_{\alpha j}^{n}\left(\bar{u}_{i, j}^{n}-\bar{u}_{i-1, j}^{n}\right)\right\}\right), \\
a_{\alpha j}^{n}=\bar{\sigma}_{\alpha j}^{n} \bar{a}_{\alpha j}^{n} / 2 . &
\end{aligned}
$$

The conditions (22) are not sufficient to maintain the BV estimates. We can get a stronger correction which implies a BV estimate stability by proceeding as follows. This is specific to the multidimensional case, since BV is not a subset of $L^{\infty}$. The analogue of Lemma 1 in one dimension yields the BV stability too.

Let $\overline{\bar{u}}^{n}$ denote the approximate solution computed after the first correction (21). On any cell $\Gamma_{i j}$, the corrected value will have the form

$$
u_{i, j}^{n}=\bar{u}_{i, j}^{n}+k_{i, j}^{n} \text {. }
$$

This way the previous correction corresponds to

$$
k_{i, j}^{n}=\tilde{k}_{i, j}^{n} \equiv \overline{\bar{u}}_{i, j}^{n}-\bar{u}_{i, j}^{n},
$$

and up to now we have no BV stability for this value. This BV stability requires a reduction of the $k_{i, j}^{n}$. In order to get it, we assume that we can find four real numbers $\alpha_{i j}^{n}, \beta_{i j}^{n}, \gamma_{i j}^{n}, \delta_{i j}^{n}$ such that

$$
\begin{aligned}
k_{i, j}^{n} & =\alpha_{i j}^{n}\left(\bar{u}_{i+1, j}^{n}-\bar{u}_{i, j}^{n}\right)=\beta_{i j}^{n}\left(\bar{u}_{i, j}^{n}-\bar{u}_{i-1, j}^{n}\right) \\
& =\gamma_{i j}^{n}\left(\bar{u}_{i, j+1}^{n}-\bar{u}_{i, j}^{n}\right)=\delta_{i j}^{n}\left(\bar{u}_{i, j}^{n}-\bar{u}_{i, j-1}^{n}\right) .
\end{aligned}
$$

This means that $k_{i, j}^{n}$ is automatically set to zero if $\bar{u}_{i, j}^{n}$ is a local extremum when compared to its neighbors $\bar{u}_{i+1, j}^{n}, \bar{u}_{i-1, j}^{n}, \bar{u}_{i, j+1}^{n}$, or $\bar{u}_{i, j-1}^{n}$. We have the following lemma. 
Theorem 2. Under the conditions

$$
\begin{aligned}
\alpha_{i j}^{n} \beta_{i j}^{n} \geq 0, & \gamma_{i j}^{n} \delta_{i j}^{n} \geq 0, \\
1-\alpha_{i j}^{n}+\beta_{i j}^{n} \geq 0, & 1-\gamma_{i j}^{n}+\delta_{i j}^{n} \geq 0
\end{aligned}
$$

we have BV stability for the scheme.

Proof. As before, we know that all operators $S(t), P_{h}$, and $Q_{h}$ preserve the total variation. It remains to prove this also for the correction operator $A_{h}$. We have

$$
\operatorname{Var}\left(u^{n} ; \mathbb{R}^{2}\right)=\left(\left|u_{i+1, j}^{n}-u_{i, j}^{n}\right|+\left|u_{i, j+1}^{n}-u_{i, j}^{n}\right|\right) h .
$$

We set

$$
\sigma_{i, j}^{n}=\operatorname{sgn}\left(\alpha_{i, j}^{n}\right)=\operatorname{sgn}\left(\beta_{i, j}^{n}\right), \quad \tau_{i, j}^{n}=\operatorname{sgn}\left(\gamma_{i, j}^{n}\right)=\operatorname{sgn}\left(\delta_{i, j}^{n}\right)
$$

and then

$$
u_{i+1, j}^{n}-u_{i, j}^{n}=\left(\bar{u}_{i+1, j}^{n}-\bar{u}_{i, j}^{n}\right)\left(1-\alpha_{i, j}^{n}+\beta_{i+1, j}^{n}\right),
$$

which leads to

$$
\left|u_{i+1, j}^{n}-u_{i, j}^{n}\right| \leq\left|\bar{u}_{i+1, j}^{n}-\bar{u}_{i, j}^{n}\right|+\sigma_{i+1, j}^{n}\left|k_{i+1, j}^{n}\right|-\sigma_{i, j}^{n}\left|k_{i, j}^{n}\right| .
$$

By using the same arguments, we get

$$
\left|u_{i, j+1}^{n}-u_{i, j}^{n}\right| \leq\left|\bar{u}_{i, j+1}^{n}-\bar{u}_{i, j}^{n}\right|+\tau_{i, j+1}^{n}\left|k_{i, j+1}^{n}\right|-\tau_{i, j}^{n}\left|k_{i, j}^{n}\right| .
$$

Now, by summing for $i, j \in \mathbb{Z}$, we get

$$
\operatorname{Var}\left(u^{n} ; \mathbb{R}^{2}\right) \leq \operatorname{Var}\left(\bar{u}^{n} ; \mathbb{R}^{2}\right),
$$

which completes the proof.

From the stability results we can state a theorem of convergence towards a weak solution. Here we denote by $u_{h}$ the approximate solution defined by

$$
u_{h}(x, t)= \begin{cases}S\left(t-t_{n-1}\right) u^{n-1} & \text { if } t_{n-1} \leq t<t_{\mu}, \\ S\left(t-t_{\mu}\right) u^{\mu} & \text { if } t_{\mu} \leq t<t_{n},\end{cases}
$$

which coincides with an exact solution of (4) on each layer $\mathbb{R}^{2} \times\left[t_{n-1}, t_{\mu}\right)$ or $\mathbb{R}^{2} \times\left[t_{\mu}, t_{n}\right)$.

Theorem 2. From the family $\left\{u_{h}\right\}$ of approximate solutions constructed by (19) with the correction operator satisfying (22) and (26), we can extract a sequence $\left\{u_{h^{\prime}}\right\}$ which converges towards a weak solution of (1) as $h$ goes to zero.

The proof is a classical one. We only sketch it. For $B_{h}$ being any of the operators $A_{h}, P_{h}, Q_{h}$, or $S(t)$, and for any $w \in \mathrm{BV}\left(\mathbb{R}^{2}\right)$, we have

$$
\left|B_{h} w\right|_{L^{\infty}} \leq|w|_{L^{\infty}}, \quad \operatorname{Var}\left(B_{h} w ; \mathbb{R}^{2}\right) \leq \operatorname{Var}\left(w ; \mathbb{R}^{2}\right)
$$

and

$$
\left|\iint_{\mathbb{R}^{2}}\left(B_{h} w-w\right) \phi d x d y\right| \leq C h^{2} \operatorname{Var}\left(w ; \mathbb{R}^{2}\right)|\nabla \phi|_{L^{\infty}},
$$

where $C$ does not depend on $h$. Then thanks to Helly's theorem, we can extract a subsequence which converges in the $L^{1}$ norm towards some function $u$ in $\operatorname{BV}\left(\mathbb{R}^{2} \times \mathbb{R}_{+}\right)$. Next, by writing that $u_{h^{\prime}}$ (from an extracted sequence) is 


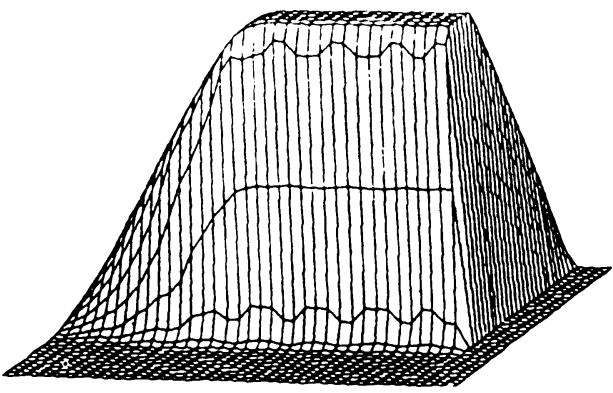

FIGURE 3

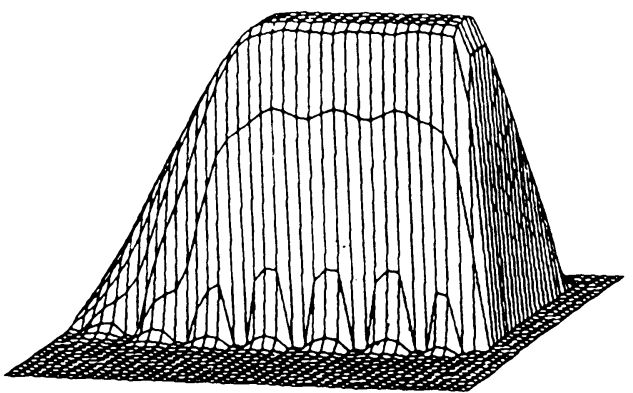

Figure 4

a weak solution on the layers $\mathbb{R}^{2} \times\left[t_{n-1}, t_{\mu}\right)$ or $\mathbb{R}^{2} \times\left[t_{\mu}, t_{n}\right)$, and using (27) to bound the jumps at each $t_{n-1}$ or $t_{\mu}$, we get that the limit $u$ is a weak solution.

To have the convergence towards the entropy solution, we assume as in [10] the coefficients $a_{\alpha j}^{n}$ and $a_{i \beta}^{n}$ to be bounded by a modulus of continuity $\omega(h)$; that is,

$$
\left|a_{\alpha j}^{n}\right| \leq \omega(h) \text { and }\left|a_{i \beta}^{n}\right| \leq \omega(h) .
$$

Then we get

Theorem 3. Suppose that the correction operator $A_{h}$ satisfies (22), (26), and (28). Then the whole family $\left\{u_{h}\right\}$ converges towards the entropy solution as $h$ goes to zero.

The proof is also a classical one. We use an approximate solution $u_{h^{\prime}}$ from any extracted sequence in the previous theorem. Since $u_{h^{\prime}}$ is an entropy solution on any layer $\mathbb{R}^{2} \times\left[t_{n-1}, t_{\mu}\right)$ or $\mathbb{R}^{2} \times\left[t_{\mu}, t_{n}\right)$, and using (27) and (28) to bound the jumps at the times $t_{n-1}$ and $t_{\mu}$, we get that the limit $u$ satisfies (4). Next, from the uniqueness of the entropy solution, we have convergence for the whole family.

We present now some computational results on the same example as before, with the same conditions. Figure 3 deals with the correction (20), (23), and Figure 4 with (24), and

$$
k_{i j}^{n}=\theta_{i j}^{n}\left(-a_{\alpha, j}^{n}+a_{\alpha-1, j}^{n}-a_{i, \beta}^{n}+a_{i, \beta-1}^{n}\right),
$$

where $\theta_{i j}^{n} \in[0,1]$ is as large as possible subject to the constraints $(26)$.

The difference between Figure 2 and Figure 4 is not significant, which means that the correction which preserves the BV norm is not really operative (that is, $k_{i, j}^{n}=0$ too often). On the other hand, Figure 3 shows a really improved result.

\section{CONCLUSIONS}

We can expect another theorem of convergence by using some results of convergence by R. DiPerna [5], or adapted to some two-dimensional cases by A. Szepessy [12]; see also [4], where the different steps of the proof are detailed. However, the hypotheses (22) and (28) seem to be not sufficient for this theorem, 
and we need to add another one, which is close to (26) in practice. In any case, a correction is needed (see [6]).

The three-dimensional case may be solved in this way also. However, the corresponding two-dimensional Riemann problem is the following one:

$$
u_{t}+\sum_{i=1}^{3}\left\{f_{i}(u)_{x_{i}}\right\}=0
$$

with

$$
u(x, 0)= \begin{cases}u_{1} & \text { if } x>0 \text { and } y>0 \\ u_{2} & \text { if } x<0 \text { and } y>0 \\ u_{3} & \text { if } x<0 \text { and } y<0 \\ u_{4} & \text { if } x>0 \text { and } y<0\end{cases}
$$

This is far more complicated, and the resulting scheme would be rather slow in practice. However, the above 2D scheme can be used to approximate the 2D Riemann problem on each 3D face in order to construct a 3D version of the scheme.

The two-dimensional scheme, mainly with the correction (22), is a relatively efficient method in terms of CPU time. This method can be adapted to systems of conservation laws as long as we are able to solve the one-dimensional Riemann solver. Many one-dimensional Riemann solvers have been generated in practice for the usual applications such as those in hydrodynamics.

This scheme allows us to work with a method which is not an alternating direction one, and no axes phenomena are expected. It may be a good investigation tool for the validation of other methods. A Lagrange-Euler version (as in [11]) can also be derived.

\section{BIBLIOGRAPHY}

1. J. P. Boris, D. L. Book, and K. Hain, Flux-corrected transport. II: Generalizations of the method, J. Comput. Phys. 18 (1975), 248-283.

2. T. Boukadida, Thèse, Bordeaux, 1988.

3. E. Conway and J. Smoller, Global solutions of the Cauchy problem for quasi-linear first-order equations in several space variables, Comm. Pure Appl. Math. 19 (1966), 95-105.

4. F. Coquel and $\mathrm{Ph}$. LeFloch, Convergence of finite difference schemes for conservation laws in several space dimensions: general theory, rapport de l'Ecole Polytechnique, no. 207, 1989.

5. R. Di Perna, Measure-valued solutions to conservation laws, Arch. Rational Mech. Anal. 88 (1985), 223-270.

6. J. Goodman and R. J. LeVeque, On the accuracy of stable schemes for 2D scalar conservation laws, Math. Comp. 45 (1985), 15-21.

7. B. Keyfitz Quinn, Solutions with shocks: an example of an $L^{1}$-contractive semigroup, Comm. Pure Appl. Math. 24 (1971), 125-132.

8. S. N. Kružkov, First order quasilinear equations in several independent variables, Math. USSR-Sb. 10 (1970), 217-132.

9. P. D. Lax, Weak solutions of nonlinear hyperbolic equations and their numerical computation, Comm. Pure Appl. Math. 7 (1954), 159-193.

10. A. Y. Le Roux, Convergence of an accurate scheme for first order quasi linear equations, RAIRO Numer. Anal. 15 (1981), 151-170. 
11. A. Y. Le Roux and P. Quesseveur, Convergence of an antidiffusion Lagrange-Euler scheme for quasi-linear equations, SIAM J. Numer. Anal. 21 (1984), 985-994.

12. A. Szepessy, Convergence of a shock-capturing streamline diffusion finite element method for a scalar conservation law in two space dimensions, Math. Comp. 53 (1989), 527-545.

Centre de Recherche en Mathematiques de Bordeaux, Universite Bordeaux 1, F-33405 Talence, France

E-mail address: AY. leroux@u-bordeaux.fr 